RESEÑAS 



\section{El Nuevo Juicio de Amparo. Guía de la reforma constitucional y la nueva ley de amparo DE EDUARDO FERRER MAC-GREGOR Y RUBÉN SÁNCHEZ GIL, MÉXICO, PORRÚA-UNAM-IMDPC, 2013.}

Juan Luis HERNÁNDEZ MACÍAS ${ }^{1}$

Exigencia de primer orden es aquella que toda revista científica tiene de propiciar la discusión de los paradigmas más álgidos que ocupan a sus respectivas comunidades del conocimiento. Cada una de las secciones en que se divide un volumen juega un papel determinado en la disertación académica, y si bien los artículos arbitrados por pares ciegos constituyen el eje rector de las publicaciones de este corte, también es cierto que el dinamismo - sobre todo de periodicidad- de una revista permite introducir otros géneros de la literatura científica.

En este sentido, es de celebrar la apertura de esta nueva sección de reseña en Ciencia Jurídica, que desde nuestra perspectiva abonará con creces a la discusión, circulación y crítica de la doctrina jurídica contemporánea. Lo anterior en el entendido de que esto no es únicamente materia de la presentación de un libro en una Universidad, congreso o sede judicial, sino que por tratarse de la retroalimentación, recomendación o descalificación argumentada de un autor hacia otro éstas debieran hacerse - en el mejor de los casos- por escrito.

La obra aquí en reseña aparece bajo el siguiente contexto: habían pasado apenas tres meses desde la publicación de la nueva Ley de Amparo el dos de abril de 2013 cuando este estudio sale de la imprenta, la variable de temporalidad juega un papel a considerar y está estrechamente ligado al hecho de que los autores hayan decidido utilizar el término guía para describir e intitular su libro. Por otra parte, la obra se presenta bajo una triple coedición, primeramente Porrúa, conocida y prestigiada editorial que se ha dedicado en buena parte de su haber a publicar la obra de los juristas mexicanos, esta obra aparece como el número 8 o de la Biblioteca Porrúa de Derecho Procesal Constitucional, misma que está bajo la dirección de uno de los autores, Eduardo Ferrer, quien se ha encargado de procurar la publicación tanto de clásicos como de la vanguardia de esta ciencia procesal constitucional en esta valiosa colección; la Universidad Nacional Autónoma de México, casa de estudios de ambos autores como estudiantes y profesores, así como Ferrer Mac-Gregor investigador de su Instituto de Investigaciones Jurídicas; y finalmente bajo el aval de seriedad académica del Instituto Mexicano de Derecho Procesal Constitucional.

La obra está dedicada a dos pilares de la construcción y concepción contemporánea del juicio de amparo, Ignacio Burgoa Orihuela y Héctor Fix-Zamudio, cuestión que no escapa de su debida atención, pues los autores - Sánchez Gil y Ferrer Mac-Gregor - son respectivamente sus discípulos académicos. En la presentación se explica que perteneciendo cada cual a escuelas que conciben el juicio de amparo de forma muy diferente, han pretendido retomar lo mejor de ambos extremos - a saber- del maestro Burgoa la identidad nacionalista del

\footnotetext{
${ }^{1}$ Universidad de Guanajuato, jlhernandezmacias@gmail.com
} 
juicio de amparo y del maestro Fix-Zamudio la incorporación del procesalismo científico y el derecho comparado a la máxima institución procesal del sistema jurídico mexicano.

Esta publicación se presenta como la primera que parte del estudio de la nueva legislación reglamentaria, ello no quiere decir que las anteriores estuvieran incompletas, deficientes o no estuvieran en aptitud de merecer comentario, simplemente que ésta es pionera al abordar ya no especulaciones sobre la regulación legislativa de las reformas de amparo y derechos humanos de junio de 2011, aspecto que - como atinadamente comentan los autores-continúa siendo insuficiente en aras de conocer y comprender el nuevo juicio de amparo. Sin embargo y a pesar de las limitaciones que los autores le reconocen a su trabajo, ha sido muy bien recibido en los diversos estadios del medio jurídico, ya bien por tratarse del primero o por la sabida trayectoria y prestigio que les ha merecido a los autores su labor de seriedad académica. Entre los últimos días de junio y los primeros de julio de 2013, el libro ha visto presentaciones en el recinto de la Suprema Corte de Justicia de la Nación (SCJN), en el Instituto de Investigaciones Jurídicas de la UNAM (IIJ-UNAM), en sedes del Poder Judicial de la Federación en todo el país y en variadas casas de estudios superiores.

El estudio está dividido en cuatro capítulos, sin tomar en cuenta la prologación del Ministro Arturo Zaldívar Lelo de Larrea - quien formara parte de la comisión designada por el Tribunal Pleno de la SCJN en 1999 para la realización del proyecto de una nueva Ley de Amparo- y una presentación que consideramos ineludible para comprender las pretensiones del material que tenemos en nuestras manos. Ya desde esta presentación conoceremos los ejes que rigen la argumentación de la obra. Por ejemplo, desde un inicio los autores dejan claro que, en pos de entender el nuevo juicio de amparo, la nueva legislación debe leerse paralelamente con la reforma de derechos humanos del 1o de junio de 2011 e igualmente con las tesis jurisprudenciales derivadas del cumplimiento de la sentencia de Rosendo Radilla Pacheco vs México de la Corte Interamericana de Derechos Humanos (CoIDH) en el expediente Varios 912/2010 en el Máximo Tribunal mexicano.

En un primer y breve capítulo los autores nos explican la turbulenta historia que sufrió un primitivo deseo de revisar la legislación de amparo hasta su tardía consumación el dos de abril de 2013, mismo que encontramos de gran utilidad para quienes pretenden iniciarse más allá del conocimiento de esta institución procesal- en la comprensión de los procesos de creación de las leyes en general como fenómeno jurídico y político, del que no únicamente participan legisladores, el relato de lo acontecido en la comisión resulta esclarecedor en este respecto. ${ }^{2}$

En un segundo apartado se hace referencia a la reforma de junio de 2011, como ya se ha dicho, los autores no conciben la idea estudiar por separado la reforma de amparo del 6 de junio y la de derechos humanos de cuatro días después. Por lo dicho, los autores se plantean varias preguntas y ensayan algunas respuestas, al menos dos merecen su mención: la primera en relación al tratamiento que los tribunales harán respecto al así llamado Control

\footnotetext{
${ }^{2}$ La comisión se conformó por los ministros Juan N. Silva Meza y Humberto Román Palacios (hoy en retiro); los magistrados César Esquinca Muñoz y Manuel Ernesto Saloma Vera; los investigadores Héctor Fix-Zamudio y José Ramón Cossío Díaz (hoy ministro de la SCJN), y; por los abogados postulantes Javier Quijano Baz y Arturo Zaldívar Lelo de Larrea (hoy ministro de la SCJN). Para una mejor explicación de lo acontecido en la comisión y su resultante proyecto, véase también: Quijano Baz, Javier, "Proyecto de la Suprema Corte de Justicia de la Nación de la Ley de Amparo", en Vega, Juan y Corzo Sosa, Edgar (coords.), Instrumentos de tutela y justicia constitucional. Memoria del VII Congreso Iberoamericano de Derecho Constitucional, México, IIJ-UNAM, 2002, pp. 455-470.
} 
de Convencionalidad, ${ }^{3}$ y la segunda relacionada con el principio pro persona inserto en el artículo primero constitucional y su relación con la polémica respectiva a si existe una relación jerárquica entre la carta magna y los tratados internacionales que contengan normas de derechos humanos. Los autores niegan tal idea y se pronuncian por la existencia de un bloque de constitucionalidad, controversia que judicialmente la SCJN resolvería meses después en la resolución de la Contradicción de Tesis 293/2011, lo cual por supuesto que no desfasa la idea descrita en la obra, por el contrario abre la puerta a que sea la comunidad académica la que continúe pronunciándose al respecto, pues igualmente la discusión al respecto de la obligatoriedad, vinculación u orientación de la jurisprudencia de la $\mathrm{CoIDH}$ — derivada del caso Gelman vs Uruguay - no parece haber encontrado su fin.

Ya entrados de lleno en materia, un tercer capítulo articula un análisis pormenorizado, si no de todos, al menos sí de la gran parte de las nuevas disposiciones reglamentarias, todas detalladas en veinte subtítulos en los que se concatena doctrina, jurisprudencia así como los puntos de vista de los autores en un análisis muy bien logrado, por poner un ejemplo, los autores hacen referencia doctrinaria al tema de la ponderación y proporcionalidad y su tímida regulación en la nueva ley, pero no se limitan a describir la forma en que - a su juicio - esta herramienta de interpretación funcionará, sino que además incorporan el tratamiento jurisprudencial que los tribunales y la SCJN le han dado de tiempo atrás. Este es, desde nuestra perspectiva, el más valioso de los capítulos de la obra y quizás el más discutible. En posteriores páginas los autores explicarán que han tratado de incorporar los criterios de jurisprudencia que podrán - a menos que los tribunales digan lo contrario- seguir aplicándole a la nueva legislación, pues como era de esperarse, muchas de las tesis emitidas anteriores al dos de abril del año corriente, quedaron desfasadas por la nueva reglamentación. Si bien algunas críticas ya se le han hecho a este estudio, la principal estriba en que una obra tan cercana a la publicación de la nueva ley no puede abordar de forma íntegra su estudio, debido a que en materia de amparo la jurisprudencia de los tribunales de la Federación constituye un aspecto ineludible para su empleo práctico, la cual aún no se ha desarrollado. Consideramos que esta crítica - lamentablemente no hecha por escrito- es bastante discutible pues volviendo sobre la denominación guía que los autores eligieron, es que se salva su pretensión. En un ejercicio de modestia, los autores se suben a los hombros de los gigantes en 754 notas al pie en 263 páginas que conforman la obra, prueba indubitable de que esta guía invita al lector a estudiar de propia cuenta los temas a profundidad en la medida que su interés se lo permita, las invitaciones y confrontaciones que se proponen al pie son referencias de primer orden, además de reflejar el trabajo de años de investigación que pretenden ser condensados en apenas unas semanas de redacción. Parafraseando a José Ramón Cossío con motivo de la presentación en el IIJ-UNAM, en este momento este libro no puede pretender ser ni un tratado ni un manual. Por lo cual consideramos que como una guía supera con creces su expectativa.

Irónica, pero atinadamente los autores ultiman la estructura de su estudio con un Balance Inicial, exponen que el ejercicio que se intentó nunca fue dar respuestas conclusivas sino plantear preguntas, ponerlas a discusión y ensayar algunas primeras y tentativas respuestas.

\footnotetext{
3 La polémica estriba en saber si realmente los tribunales mexicanos pueden hacer control convencional o sencillamente hacen control constitucional con reenvío a la legislación de las convenciones internacionales, y son así los tribunales internacionales los que tienen el monopolio del control convencional. La discusión se reduce a una vaguedad terminológica.
} 
Celebran la llegada de la nueva legislación a pesar de las deficiencias que consideran a lo largo de las páginas del libro.

La obra por sí sola invita al estudio, no sólo a estudiantes, sino a todos los agentes que tendrán delegada la tarea de poner en marcha este nuevo juicio de amparo, ya en las últimas páginas los autores aseverarán: "es imposible que haya buena administración de justicia cuando la ejercen jueces malos, por buenas que sean sus leyes", al tiempo que invitan a las universidades del país a modificar sus planes de estudio en razón de la nueva ley, idea que es muy discutible, una educación jurídica integral basada en legislación, jurisprudencia y doctrina no debería sino sólo cambiar uno de sus múltiples objetos de estudio. Nada impedía estudiar interés legítimo, declaratoria general de inconstitucionalidad o suspensión con apariencia de buen derecho sin que existiera una ley que las regulara, todavía actualmente nada nos impide incorporar todo aquello que la ley omitió o reguló deficientemente, justamente esta parte es la que otorga mérito a la obra en comento.

Una investigación seria y completa es la que ponen al escrutinio de la comunidad jurídica Eduardo Ferrer Mac-Gregor, investigador consolidado y juez de la CoIDH y Rubén Sánchez Gil, abogado postulante formado en la academia y cada vez más un referente sobresaliente en materia de derecho procesal constitucional. 